\title{
BMJ Open Financial conflicts of interest of clinicians making submissions to the pan-Canadian Oncology Drug Review: a descriptive study
}

Joel Lexchin ${ }^{\odot}$

To cite: Lexchin J. Financial conflicts of interest of clinicians making submissions to the pan-Canadian Oncology Drug Review: a descriptive study. BMJ Open 2019;9:e030750. doi:10.1136/ bmjopen-2019-030750

- Prepublication history and additional material for this paper are available online. To view these files, please visit the journal online (http://dx.doi. org/10.1136/bmjopen-2019030750).

Received 29 March 2019

Revised 2 June 2019

Accepted 10 June 2019

\section{Check for updates}

(C) Author(s) (or their employer(s)) 2019. Re-use permitted under CC BY-NC. No commercial re-use. See rights and permissions. Published by BMJ.

School of Health Policy \& Management, York University, Toronto, Ontario, Canada

Correspondence to Dr Joel Lexchin; jlexchin@yorku.ca

\section{ABSTRACT}

Objectives This study examines financial conflict of interest (FCOI) of clinicians who made submissions to the panCanadian Oncology Drug Review (pCODR), the arm of the Canadian Agency for Drugs and Technology in Health that recommends whether oncology drug indications should be publicly funded. Final reports from pCODR published between October 2016 and February 2019 were examined.

Design Descriptive study.

Data sources Website of pCODR.

Interventions None.

Primary and secondary outcomes The primary outcome is the number of submissions declaring FCOI. Secondary outcomes are the number of times where clinicians agreed and disagreed with preliminary recommendation from pCODR and the association between the distribution of individual clinicians' FCOI and pCODR's funding recommendations.

Results There were 46 drug indication reports from pCODR. Clinicians made 261 submissions. Clinicians declared they received payments from companies 323 times and named 38 different companies making those payments a total of 500 times. Financial conflicts with drug companies were declared in $176(66.3 \%)$ of all submissions. In 21 (45.7\%) of the 46 drug indications, $50 \%$ or more of the clinicians had a conflict with the company making the drug. Clinicians commented on 37 preliminary recommendations. In all 25 where pCODR recommended funding or conditional funding, the clinicians either agreed or agreed in part. pCODR recommended that the drug indication not be funded 12 times and 9 times clinicians disagreed with that recommendation. The distribution of clinician responses was statistically significantly different depending on whether $\mathrm{pCODR}$ recommended funding/conditional funding or do not fund $p<0.0001$ (Fisher exact test). The distribution of clinicians' FCOI differed depending on whether the recommendation was fund/conditional fund or do not fund $\mathrm{p}=0.027$ (Fisher exact test).

Conclusion Financial conflicts with pharmaceutical companies are widespread among experts making submissions to the PCODR.

\section{INTRODUCTION}

Canada has no national drug formulary and as a result, the federal, provincial and territorial governments (except for Quebec) cooperate
Strengths and limitations of this study

- This is the first study to examine financial conflict of interest (FCOI) of clinicians making submissions to the pan-Canadian Oncology Drug Review (pCODR).

- All clinician submissions were evaluated rather than just a sample of submissions.

- Results only apply to oncology clinicians making voluntary, unsolicited submissions to pCODR

- No independent checking about accuracy of FCOI declarations.

- No data available to determine if FCOI affect clinicians' views about funding.

through the Canadian Agency for Drugs and Technology in Health (CADTH) to make recommendations about whether to fund unique drug-indication combinations. Specifically, the pan-Canadian Oncology Drug Review (pCODR), an arm of CADTH has been doing this for oncology products starting in January 2012. ${ }^{1}$ Briefly, pCODR accepts applications from manufacturers and drug plans and then uses an expert panel ${ }^{2}{ }^{3}$ that considers the clinical evidence, plus input from manufacturers and voluntary, unsolicited submissions from clinicians and patient groups in making its recommendations about whether the plans should list drugs for specific indications.

Since October 2016, pCODR has published input from registered clinicians defined as practising oncologists or physicians who treat cancer patients, oncology pharmacists and oncology nurses. "Oncologists or physicians who treat cancer patients can provide their input as an individual submission or jointly in a group submission. Oncology pharmacists and oncology nurses provide invaluable information on drug preparation and administration, and are eligible to provide input as part of a joint submission with a lead oncologist'. ${ }^{4}$ Part of the process of registering is completing a financial conflict of interest (FCOI) form. ${ }^{5}$ 
Once registered, clinicians receive notifications via email of all upcoming reviews at pCODR and can voluntarily make unsolicited submissions. The email notification has information pertaining to the drug and indication under review, the link to the clinician input template, and the deadline date for submitting input.

In the USA, FCOI is associated with the voting patterns of members of Food and Drug Administration (FDA) advisory committees ${ }^{6}$ but there has not been any analysis of FCOIs of clinicians' input into funding recommendations. This study was undertaken to examine the distribution of FCOIs of clinicians making inputs into the pCODR process.

\section{METHODS}

\section{Source of data}

Reports from pCODR are available at https://www.cadth. $\mathrm{ca} / \mathrm{pcodr} /$ find-a-review. All reports were included if a final recommendation had been issued between October 2016 and 22 February 2019 and if they included a submission from one or more clinicians. Applications from manufacturers where they were requesting a reconsideration of a previous decision or where they were requesting funding for a different drug indication for the same drug were included and treated as separate applications. Besides allowing clinicians to make inputs to pCODR, they are also allowed to comment on preliminary decisions.

\section{Information extracted from pCODR reports}

From each report the following information was extracted: generic and brand name of drug, indication, company manufacturing the drug, preliminary and final recommendations about funding and whether the clinicians agreed, agreed in part (agreed with the overall recommendation but requested modifications, for example, expand patient group that should be covered) or disagreed with the preliminary recommendation about funding-fund, fund based on conditions being fulfilled (eg, the drug being cost-effective or budgetary effects being taken into consideration) and do not fund.

FCOI forms contain the name of the clinicians and ask them to declare payments received within the previous 2 years for one or more of 10 types of activities: advisory role (advisory board and/or health technology advice), conference attendance, gifts, honoraria, royalties, programme or operating funding (eg, website), research/educational grants, sponsorship of events, travel grants and other. In addition, clinicians need to give the names of companies making the payments and the amounts of the payments. Clinicians also need to list whether they have received or are in possession of stocks or options of more than $\$ 10000$ (excluding mutual funds) for organisations that may have a direct or indirect interest in the drug under review and whether they have personal or commercial relationships either with a drug or health technology manufacturer (including such manufacturer's parent corporation, subsidiaries, affiliates and associated corporations) or other interest groups. Information on all of these categories was extracted and put into an Excel spreadsheet. The status of the clinician making the submission, that is, physician, pharmacist and so on, is not contained in the FCOI statement and the amounts of money received from each company is blacked out.

Information was extracted by the author in March 2019 and verified by CO, a retired general practitioner. Disagreements were resolved by discussion.

\section{Analyses of information from pCODR reports}

Counts were made of the following: number of individual drug-indication reports from pCODR along with the number of clinicians making submissions per drug-indication, how many drug companies each clinician had a conflict with, whether they had a conflict with the company making the drug, a conflict with another drug company or had no declared conflicts with companies and the number of paid activities for drug companies each clinician reported when they made a submission. Based on this data, the number of submissions where a majority of clinicians had a conflict with any drug company and a conflict with the company making the drug was calculated. In addition, the number of different submissions from each clinician was totaled. Finally counts were made of the number of times a clinician reported stocks or options and personal or commercial relationships.

If a clinician or group of clinicians made a comment about the preliminary recommendation-agree, agree in part, disagree-the distribution of the type of comment was compared for each of the three possible funding recommendations. Comments were sometimes made by a group of clinicians and in those cases, it was not possible to identify specific people to determine their FCOI. Where clinicians submitting comments were named, their FCOI (with the submitting company, with another company or no FCOI) were recorded and the distribution of FCOI was compared depending on whether the recommendation was fund/conditional funding or do not fund.

\section{Statistics}

Agreement between views of clinicians about preliminary recommendations and the recommendations from pCODR and the distribution of the types of clinicians' FCOI between different funding recommendations were both analysed using Fisher's exact test. Prism V.7.0d for Macintosh (GraphPad Software) was used for statistical testing.

\section{Patient and public involvement \\ No patients were involved in this study.}

\section{RESULTS}

There were reports for 46 drug indications and clinicians made 261 submissions for these 46 drug indications (an additional 10 reports did not include clinician 
Table 1 Number (per cent) of clinicians declaring financial conflicts with companies and payments for activities

\begin{tabular}{llcc}
\hline Status of conflict declared by clinician & $\begin{array}{l}\text { Number of clinician } \\
\text { submissions (per cent } \\
\text { all 261 submissions) }\end{array}$ & $\begin{array}{l}\text { Number of times } \begin{array}{l}\text { Number of mentions of } \\
\text { payments declared for companies that clinicians } \\
\text { activities (per cent all } \\
\text { payments) }\end{array} \\
\text { had conflict with (per cent } \\
\text { all mentions) }\end{array}$ \\
\hline $\begin{array}{l}\text { Number of submissions with conflicts declared } \\
\text { Conflict with company marketing drug }\end{array}$ & $173(66.3)$ & 323 & 500 \\
\hline $\begin{array}{l}\text { Conflict with another company } \\
\text { Conflict declared but company not named }\end{array}$ & $52(19.9)$ & $232(71.8)$ & $345(69.0)$ \\
$\begin{array}{l}\text { Number of submissions declaring no conflict } \\
\text { Number of submissions where conflict declaration } \\
\text { missing }\end{array}$ & $78(29.9)$ & $10(3.1)$ & $155(31.0)$ \\
\hline
\end{tabular}

submissions). Financial conflicts with drug companies were declared in $176(66.3 \%)$ of all submissions; 119 times $(45.6 \%)$ conflicts were with the company that made the drug under consideration; 52 times (19.9\%) with another company and in five cases $(1.9 \%)$ the name of the company was missing. In 78 cases $(29.9 \%)$, clinicians did not declare any conflict, and in seven cases $(2.7 \%)$ it was not known if a conflict existed because all of the information was missing (table 1). In 33 out of the $46(71.7 \%$ ) drug indications, $50 \%$ or more of the clinicians making a submission had a conflict with one or more drug companies and in $21(45.7 \%)$ of the $46,50 \%$ or more of the clinicians had a conflict with the company making the drug (online supplementary file 1).

Clinicians declared payments 323 times in the 10 different categories of activities; 232 (71.8\%) were declared by clinicians with a conflict with the company making the drug and $81(25.1 \%)$ were declared by clinicians who had conflicts with other companies (table 1). Clinicians had conflicts with a mean of 2.9 drug companies and performed a mean of 1.9 activities for which they were paid. Payments for serving in an advisory role were declared 151 times and for the receipt of honoraria 88 times. Payments for other types of activities occurred less often (table 2). Some clinicians declared receiving payments for different types of activities for different companies in different submissions.

The clinicians declaring conflicts named 38 different drug companies a total of 500 times ranging from Merck with 57 mentions to 8 companies with a single mention (online supplementary file 2). There were five declarations of stock ownership, all by the same person but information on this topic was missing in 31 out of 261 (11.9\%) submissions. There were four declarations of a personal or commercial relationship and information was missing 33 times out of 261 submissions (12.6\%). Individual clinicians made between 1 and 10 separate submissions (table 3).

There were 46 preliminary recommendations from pCODR (33 fund or conditional funding and 13 do not fund) and clinicians commented on 37 of these (25 fund or conditional funding and 12 do not fund). In all 25 cases where pCODR recommended funding or conditional funding the clinicians either agreed or agreed in part. Twelve times pCODR recommended that the drug-indication not be funded and nine times clinicians disagreed with that recommendation, in one case they agreed and in two cases they agreed in part (table 4 ). The distribution of clinician responses was statistically significantly different depending on whether pCODR recommended funding/conditional funding or do not fund $\mathrm{p}<0.0001$ (Fisher exact test). In one case, the pCODR changed its preliminary do not fund recommendation to a final conditional funding recommendation as a result of a re-examination of the efficacy and safety information based on the feedback pCODR received from the company, clinicians and two patient groups (https:// www.cadth.ca/sites/default/files/pcodr/pcodr_venetoclax_venclexta_cll_fn_rec.pdf).

There were 40 clinicians who provided comments on 13 preliminary recommendations where it was possible to determine their FCOI. (Comments on the other 24 preliminary recommendations were made by a group of clinicians and names of individuals were not provided.) When the recommendation was fund or fund with conditions or criteria the majority of clinicians (18 out of 27) had no FCOI whereas when the recommendation was do not fund, the plurality of clinicians (6 out of 13) had an

Table 2 Number of different types of activities for which payments received

\begin{tabular}{llllllllll}
\hline $\begin{array}{l}\text { Type of } \\
\text { activity }\end{array}$ & $\begin{array}{l}\text { Advisory } \\
\text { role }\end{array}$ & $\begin{array}{l}\text { Conference } \\
\text { attendance }\end{array}$ & Gifts & Honoraria & $\begin{array}{l}\text { Programme } \\
\text { or operating } \\
\text { funding }\end{array}$ & $\begin{array}{l}\text { Research/ } \\
\text { educational } \\
\text { grants }\end{array}$ & Royalties & $\begin{array}{l}\text { Travel } \\
\text { grants }\end{array}$ & $\begin{array}{l}\text { Sponsorship } \\
\text { of events }\end{array}$ \\
Other
\end{tabular}


Table 3 Number of individual submissions per clinician

\begin{tabular}{lc} 
Number of clinicians & Number of individual submissions \\
\hline 69 & 1 \\
28 & 2 \\
11 & 3 \\
4 & 4 \\
8 & 5 \\
1 & 6 \\
1 & 7 \\
1 & 8 \\
2 & 10 \\
\hline
\end{tabular}

FCOI with the submitting company (table 5). The distribution of FCOI of clinicians depending on the type of preliminary recommendation was statistically significantly different, $\mathrm{p}=0.027$ (Fisher exact test).

\section{DISCUSSION}

The results of this study show that two-thirds of the clinicians who make submissions to the pCODR have FCOI with one or more pharmaceutical companies and almost half have FCOI with the company making the product that is being considered for public funding. The amount of money that clinicians received for their activities on behalf of the companies is not known as that information is blacked out on their FCOI declaration forms. In over $70 \%$ of the drug-indications being reviewed by the pCODR, the majority of clinicians making submissions had conflicts. The largest number of activities for which physicians were paid was serving in an 'advisory role', but exactly what this means is not clarified in the FCOI documents that are made public and it is possible that this term was interpreted differently by individual clinicians.

The level of COI revealed in this study is greater than that reported in a 2015 survey of Canadian physicians, where $46 \%$ said that they had been retained by a pharmaceutical company in some capacity at some point in their career. ${ }^{7}$ It is also substantially larger than the level of FCOI of people serving on FDA advisory committees. These are committees convened by the FDA to vote on whether the

Table 4 Clinician response to preliminary recommendation from pan-Canadian Oncology Drug Review

\section{Preliminary recommendation from pan- Canadian Oncology Drug Review}

\begin{tabular}{llll}
\cline { 2 - 4 } $\begin{array}{l}\text { Response from } \\
\text { clinicians }\end{array}$ & Fund & $\begin{array}{l}\text { Fund with } \\
\text { conditions or } \\
\text { criteria }\end{array}$ & $\begin{array}{l}\text { Do not } \\
\text { fund }\end{array}$ \\
\hline Agrees & 1 & 17 & 1 \\
Agrees in part & 0 & 7 & 2 \\
Disagrees & 0 & 0 & 9 \\
\hline
\end{tabular}

$\mathrm{P}<0.0001$, Fisher exact test.
Table 5 Association between conflict of clinicians and preliminary funding recommendation

\begin{tabular}{llll}
\hline & $\begin{array}{l}\text { Fund/fund with conditions or } \\
\text { criteria }\end{array}$ & \\
\hline & $\begin{array}{l}\text { Conflict } \\
\text { with } \\
\text { company } \\
\text { making } \\
\text { drug }\end{array}$ & $\begin{array}{l}\text { Conflict } \\
\text { with } \\
\text { another } \\
\text { company }\end{array}$ & No conflict \\
\hline Agree/agree in part & 4 & 5 & 18 \\
\hline & Do not fund & \\
\hline & $\begin{array}{l}\text { Conflict } \\
\text { with } \\
\text { company } \\
\text { making } \\
\text { drug }\end{array}$ & $\begin{array}{l}\text { Conflict } \\
\text { with } \\
\text { another } \\
\text { company }\end{array}$ & \\
\hline & 6 & 4 & No conflict \\
\hline Disagree & 6 & & 3 \\
\hline
\end{tabular}

$P=0.027$, Fisher exact test.

FDA should approve new drug applications. An analysis of 379 meetings held over 15 years by 15 committees found that the median level of meeting 'conflictedness' (percentage of individuals with a reported FCOI) was around $13 \%$ (range $2 \%-29 \%$ ). On average, committees reported that half of their meetings were attended by at least one person with a financial conflict. ${ }^{6}$

An additional issue that this study identified was that there is missing information in a substantial number of FCOI declarations. Statements about stock ownership were not completed $11.9 \%$ of the time and those about a personal or commercial relationship were not completed $12.6 \%$ of the time. These omissions raise the question about whether these declarations are just pro forma, that is, a piece of paper to be filled out and then ignored by the pCODR. Moreover, there is evidence that declarations about FCOI are often omitted in medical journal articles, ${ }^{89}$ in clinical practice guidelines ${ }^{10} 11$ and among people presenting at conferences. ${ }^{12}$

Two important questions are whether the conflicts held by the clinicians influenced their view about the drug-indication being considered and whether the conflicts influenced the final decision by the pCODR. pCODR does not publish the submissions from the clinicians but summarises them in its reports and does not necessarily attribute views to individual people or individual groups in the case where more than one individual or group makes a submission. Therefore, when there are submissions from more than one individual clinician or more than one group of clinicians it is generally not possible to link views about a drug-indication (positive, neutral, negative) to the FCOIs. However, $75 \%$ of the time when the preliminary recommendation of the pCODR was not to fund the drug-indication clinicians making submissions disagreed with the decision and most of the clinicians who disagreed with the recommendation declared an FCOI with the company making the product. These 
finding suggest that FCOI did determine clinicians' views about the product. This suggestion needs to be tempered by a couple of points. First, clinicians may have held favourable views about the drug-indication before their relationship with a drug company started. Second, there can be a legitimate argument about whose views of the drug-indication were more accurate, those of the clinicians or those of the pCODR.

As to whether or not the clinicians had an influence on the final recommendation of the pCODR, it may be relevant that the pCODR only changed its recommendation from do not fund to fund with conditions in one out of the nine cases where the clinicians disagreed with the preliminary decision. Removing all of the FDA advisory committee members with conflicts would have produced margins less favourable to the drug being considered in the majority of meetings, but this would not have changed whether the majority favoured or opposed the drug. ${ }^{13}$

In Australia, the Pharmaceutical Benefits Advisory Committee (PBAC) serves somewhat the same role as the pCODR. ${ }^{14}$ In making submissions to the PBAC, companies are able to recruit experts to provide an opinion about their drug and the sponsors have to provide a signed statement from each expert about their FCOIs. ${ }^{15}$ However, the FCOI documents from these experts are not publicly available so their degree of FCOI cannot be compared with that of experts giving input to pCODR.

The literature about whether disclosure of FCOI affects trust in individual doctors, the pharmaceutical industry and the healthcare system in general is mixed. A systematic review about the impact of disclosing financial ties in research and clinical care, found that patients believe that these influenced professional behaviour, decreased the quality of research evidence and should be disclosed. ${ }^{16}$ More recently, one study reported that when patients believed that a gift relationship existed, they had lower levels of physician trust and higher rates of healthcare system distrust. ${ }^{17}$ While the level of payments affected perceptions of honesty and fidelity in individual physicians, viewing an online disclosure database did not affect patients' trust ratings for the medical profession or the pharmaceutical industry. ${ }^{18}$ Patient attitudes about FCOI in cancer research seems to be more forgiving. Most patients in cancer-research trials were not worried about FCOI between researchers and drug companies and would still have enrolled in the trial if they had known about these relationships. ${ }^{19}$ The only Canadian study on patients' attitudes found that public opinions on physician-pharmaceutical industry interactions differ depending on the scenario but suggested a significant level of concern regarding interactions involving direct financial benefit to physicians. ${ }^{20}$ Whether the conflicts held by clinicians leads to a public perception that the pCODR process is biased is a question that this current study cannot answer but should be the subject of further research.

\section{Limitations}

These results about the presence of conflicts only apply to clinicians making voluntary, unsolicited submissions about funding oncology drugs and the distribution of conflicts among other clinicians treating cancer who did not make a submission may be different. Similarly, the results may not apply to clinicians treating other diseases. Clinicians did not comment on 9 out of 46 preliminary recommendations. Whether they agreed with the final recommendation from PCODR is not known although it seems unlikely that they would have changed their views between the preliminary and final recommendations. FCOI declarations were not independently checked to see if there were undisclosed FCOI. The main strength of this study is that it looked at the entire population of recommendations from pCODR where clinicians made a submission about the drug-indication being considered.

\section{CONCLUSION}

Conflicts with pharmaceutical companies are widespread among experts making submissions to the pCODR. Information about how much experts are reimbursed for the activities that they undertake on behalf of companies is not disclosed by pCODR and attributing views to individual clinicians or groups of clinicians cannot be done since only summaries of the submissions are published. pCODR should publish full submissions, the exact amounts that clinicians receive and the names of companies making each of the payments, in order to help determine any association between payments and clinicians' views. pCODR could also consider specifically asking clinicians without any FCOI to make submissions.

Acknowledgements - Catherine Oliver verified data extraction.

Contributors $\mathrm{JL}$ came up with the idea for this study, gathered and analysed the data and wrote the manuscript.

Funding The authors have not declared a specific grant for this research from any funding agency in the public, commercial or not-for-profit sectors.

Competing interests In 2016-2019, JL was a paid consultant on two projects: one looking at developing principles for conservative diagnosis (Gordon and Betty Moore Foundation) and a second deciding what drugs should be provided free of charge by general practitioners (Government of Canada, Ontario Supporting Patient Oriented Research Support Unit and the St Michael's Hospital Foundation). He also received payment for being on a panel at the American Diabetes Association, for a talk at the Toronto Reference Library, for writing a brief for a law firm and from the Canadian Institutes of Health Research for presenting at a workshop on conflict of interest in clinical practice guidelines. He is currently a member of research groups that are receiving money from the Canadian Institutes of Health Research and the Australian National Health and Medical Research Council. He is a member of the Foundation Board of Health Action International and the Board of Canadian Doctors for Medicare.

Patient consent for publication Not required.

Ethics approval All data were publicly available and therefore ethics approval was not necessary.

Provenance and peer review Not commissioned; externally peer reviewed.

Data sharing statement Data are available in a public, open access repository.

Open access This is an open access article distributed in accordance with the Creative Commons Attribution Non Commercial (CC BY-NC 4.0) license, which permits others to distribute, remix, adapt, build upon this work non-commercially, 
and license their derivative works on different terms, provided the original work is properly cited, appropriate credit is given, any changes made indicated, and the use is non-commercial. See: http://creativecommons.org/licenses/by-nc/4.0/.

\section{REFERENCES}

1. CADTH. About the pan-Canadian Oncology Drug Review (pCODR): CADTH. 2019 https://www.cadth.ca/pcodr/about-pcodr

2. CADTH. pCODR expert review committee terms of reference. 2016 https://www.cadth.ca/sites/default/files/pcodr/The\%20pCODR\% 20Expert\%20Review\%20Committee\%20\%28pERC\%29/pcodr expertreviewcom tor.pdf

3. CADTH. CADTH Common Drug Review. Procedure for the CADTH Common Drug Review. 2014 https://www.cadth.ca/sites/default/files/ cdr/process/Procedure_for_CADTH_CDR.pdf

4. CADTH. pCODR clinician input and feedback: CADTH. 2019 https:// cadth.ca/pcodr/clinician-input-and-feedback

5. CADTH. Frequently asked questions: clinician input and feedback for the CADTH pCODR program: CADTH;. no date https://cadth. ca/sites/default/files/pcodr/pCODR\%27s\%20Drug\%20Review\% 20Process/pCODR_ClinicianlnputFeedbackFAQ.pdf

6. Pham-Kanter G. Revisiting financial conflicts of interest in FDA advisory committees. Milbank Q 2014;92:446-70.

7. Leslie C. Relationshiip between MDs and pharma changing. The Medical Post 2015.

8. Weinfurt KP, Seils DM, Tzeng JP, et al. Consistency of financial interest disclosures in the biomedical literature: the case of coronary stents. PLoS One 2008;3:e2128.

9. Rasmussen K, Schroll J, Gøtzsche PC, et al. Under-reporting of conflicts of interest among trialists: a cross-sectional study. J R Soc Med 2015;108:101-7.
10. Moynihan R, Lai A, Jarvis $\mathrm{H}$, et al. Undisclosed financial ties between guideline writers and pharmaceutical companies: a cross-sectional study across 10 disease categories. BMJ Open 2019;9:e025864.

11. Khan R, Scaffidi MA, Rumman A, et al. Prevalence of financial conflicts of interest among authors of clinical guidelines related to high-revenue medications. JAMA Intern Med 2018;178:1712-5.

12. Choo KJ, Yi PH, Burns R, et al. Variable reporting by authors presenting arthroplasty research at multiple annual conferences. $J$ Arthroplasty 2017;32:315-9.

13. Lurie P, Almeida CM, Stine N, et al. Financial conflict of interest disclosure and voting patterns at Food and Drug Administration Drug Advisory Committee meetings. JAMA 2006;295:1921-8.

14. Pharmaceutical Benefits Scheme. Pharmaceutical Benefits Advisory Committee (PBAC) membership: Australian Government Department of Health. 2019 http://www.pbs.gov.au/info/industry/listing/ participants/pbac

15. Pharmaceutical Benefits Scheme. Guidelines: Appendix 1 expert opinion: Australian Government Department of Health. 2019 https:// pbac.pbs.gov.au/appendixes/appendix-1-expert-opinion.html

16. Licurse A, Barber $\mathrm{E}$, Joffe $\mathrm{S}$, et al. The impact of disclosing financial ties in research and clinical care: a systematic review. Arch Intern Med 2010;170:675-82

17. Grande D, Shea JA, Armstrong K. Pharmaceutical industry gifts to physicians: patient beliefs and trust in physicians and the health care system. J Gen Intern Med 2012;27:274-9.

18. Hwong AR, Sah S, Lehmann LS. The effects of public disclosure of industry payments to physicians on patient trust: a randomized experiment. J Gen Intern Med 2017;32:1186-92.

19. Hampson LA, Agrawal M, Joffe S, et al. Patients' views on financial conflicts of interest in cancer research trials. N Engl J Med 2006;355:2330-7.

20. Holbrook A, Lexchin J, Pullenayegum E, et al. What do Canadians think about physician-pharmaceutical industry interactions? Health Policy 2013;112:255-63. 